\title{
Petrous Apex
}

National Cancer Institute

\section{Source}

National Cancer Institute. Petrous Apex. NCI Thesaurus. Code C62643.

The pyramidal shaped part of the temporal bone located at the base of the skull between the sphenoid and occipital bones. 